\title{
Involving people with lived experience in research on suicide prevention
}

\author{
Sarah MacLean MA, Craig Mackie MA, Simon Hatcher MBBS MD
}

Cite as: CMAJ 2018;190(Suppl 1):S13-S14. doi: 10.1503/cmaj.180485

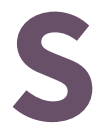

uicide is one of the leading causes of death in Canada, with about 10 Canadians dying by suicide each day. ${ }^{1}$ Men represent nearly two-thirds of those who die by suicide after an index episode of self-harm. ${ }^{2}$ However, interventions for these men have had modest effects, with poor engagement with outpatient treatment. ${ }^{3}$ The aim of the BEACON Study was to partner with people with lived experience to design an innovative intervention for self-harm specifically designed for men, and assist with the development of a recruitment strategy to better engage men who present to the emergency department with self-harm.

The BEACON Study is a cluster randomized controlled trial (ClinicalTrials.gov NCT03489382 and NCT03473535) in which 25 emergency departments in Ontario were randomized to receive one of the following for men who present for an episode of selfharm: a blended therapy service, in which face-to-face therapy is supplemented with a smartphone application, or treatment as usual. Randomization was based on data obtained from ICES. We calculated an intracluster correlation coefficient of 0.01 and an interperiod correlation of 0.008 . We considered a minimum relative reduction of $50 \%$ in the repetition rate to be both clinically important and achievable.,5 Given an average cluster size of 80 men and a coefficient of variation of cluster sizes of 30\%, 10 intervention and 15 control hospitals achieve $90 \%$ power to detect the minimum important difference.

Men who present to an intervention site will be offered an opportunity to receive seven sessions of face-to-face psychotherapy over six months, supplemented by one year of access to a smartphone application developed specifically for men who selfharm. The trial will assess outcomes at the cluster level, with a composite primary outcome measure of the proportion of suicides or self-harm repeat presentations by men within one year of study launch. An embedded cohort study will assess participant outcomes, with a primary outcome of changes in suicidality over a one-year period.

Our approach to patient engagement was twofold: we included patient partners as co-investigators on the study, and we sought to create a community of people with lived experience interested in research on suicide prevention. To achieve this, we approached service users with whom we were already in contact to either join the study as co-investigators or refer other people

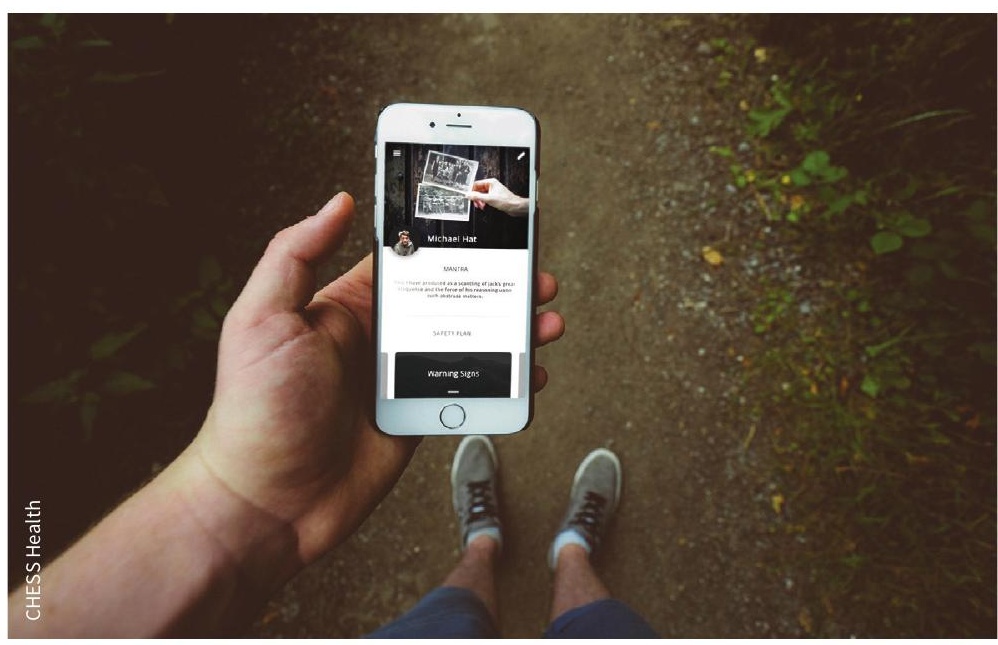

\section{KEY POINTS}

- "Testament" is a powerful and important tool for meaningful patient engagement.

- People with lived experience of mental illness and researchers need training in how to collaborate effectively.

- It is essential to create new research processes that include patient partners rather than trying to fit service users into existing structures.

with lived experience. The two service users we partnered with have been involved in all aspects of the BEACON Study, from the granting phase to the development of the study protocol to participation in the study's steering committee. We attempted to establish a Service User and Caregiver Research Interest Group (SUCRIG) in suicide prevention. The objective was to create a self-sustaining committee in which people with lived experience were engaged in research not as participants but as instigators of research. This was only partly successful, as service users did not respond well to formal meetings with minutes and agendas.

In discussing these challenges, SUCRIG committee members (total of 28 participants) emphasized the importance of providing training opportunities for potential members and the need to 
focus on specific tasks for the group. To address this, we held REFLECT 2017, a three-day conference in which we invited service users and researchers to discuss how the two groups could collaborate on suicide-prevention research. The event touched on several themes related to meaningful patient engagement, such as how to include diverse and sometimes extreme views; how researchers with lived experience might approach this dual role; what training researchers and people with lived experience need to work together; how to handle compensation; and when to approach individuals rather than patient organizations. Researchers wanted to know how to engage service users in a meaningful way, while also creating a safe environment for people with lived experience to ensure that they felt heard in a respectful way. The conference resulted in a recommended practice guide, which is currently in development, on involving people with lived experience in research on suicide prevention.

A key lesson learned from the patient-engagement strategies implemented via the BEACON Study was the importance of developing new processes for patient-oriented research studies rather than trying to fit patient engagement within existing research practices. This highlights the need to create a safe environment where patients are able to share their personal experiences through the power of "testament." We refer to it as testament because it appears to be both something to treasure, "something sacred," as well as a record of the important events that enabled someone to gain access to the research environment. To this end, one of the ways we now do things differently is to create time for people with lived experience to offer testament at research meetings when they attend for the first time. We have also learned that patient engagement within the field of mental health presents a number of unique challenges not necessarily experienced in other disciplines. For instance, additional support must be provided for patient partners to withdraw or rejoin the study as needed as they move along the continuum of mental wellness.

The BEACON Study will provide a case example of how to conduct patient-oriented research in the field of suicide prevention. Through this study, we have been provided with an opportunity to enhance the experience of mental health care for men who present to the emergency department with self-harm, a high-risk and chronically underserved population, in a way that is meaningful to patients. However, we have also learned many valuable lessons about how to provide a safe environment to engage patient partners in this field of research.

\section{References}

1. Public Health Agency of Canada. Suicide in Canada: current context. Available: http://healthycanadians.gc.ca/publications/healthy-living-vie-saine/suicide -canada-infographic/alt/infographic-infographique-eng.pdf (accessed 2018 Oct. 2).

2. Hawton K, Harriss L, Zahl D. Deaths from all causes in a long-term follow-up study of 11,583 deliberate self-harm patients. Psychol Med 2006;36:397-405.

3. Hawton K, Witt KG, Taylor Salisbury TL, et al. Psychosocial interventions for self-harm in adults. Cochrane Database Syst Rev 2016;(5):CD012189.

4. Milnes D, Owens D, Blenkiron P. Problems reported by self-harm patients: perception, hopelessness, and suicidal intent. J Psychosom Res 2002;53:819-22.

5. Canadian coroner and medical examiner database: annual report - 2006 to 2008. Cat no 82-214-X. Ottawa: Statistics Canada and Public Health Agency of Canada; 2012. Available: www150.statcan.gc.ca/n1/pub/82-214-x/82-214 -x2012001-eng.pdf (accessed 2017 May 12).

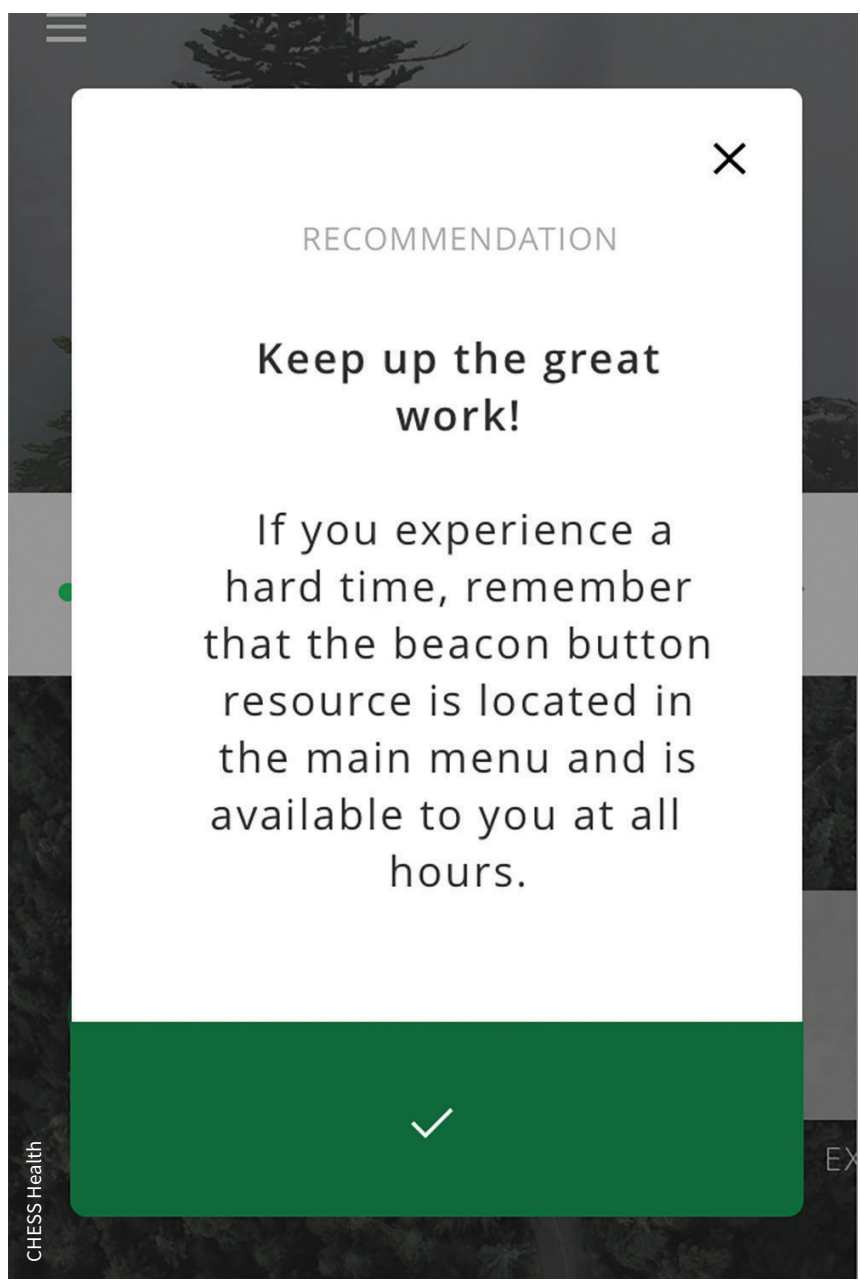

More information on this project is available at www.ossu.ca/ IMPACTAwards.

Competing interests: Simon Hatcher reports a grant from OSSU (the Ontario SPOR [Strategy for Patient-Oriented Research] SUPPORT [Support for People and Patient-Oriented Research and Trials] Unit). No other competing interests were declared.

This article was solicited and has been peer reviewed.

Affiliations: Clinical Epidemiology Program (MacLean, MacKie), The Ottawa Hospital Research Institute; Patient partner (MacKie); Department of Psychiatry (Hatcher), University of Ottawa, Ottawa, Ont.

Contributors: All of the authors contributed to drafting and revising the manuscript, gave final approval of the version to be published, and agreed to be accountable for all aspects of the work.

Funding: Funding is provided by OSSU, which is supported by the Canadian Institutes of Health Research and the Government of Ontario.

Acknowledgements: The authors acknowledge the hard work of all BEACON Study co-investigators, especially those with lived experience. Their contributions to the development of the study protocol and patient-engagement strategies have been invaluable. For a complete list, please refer to the following website: www.hatchingideashub.com/ beacon/cluster-rct.

Correspondence to: Simon Hatcher, shatcher@uottawa.ca 